\section{Case Reports in Neurology}

Case Rep Neurol 2021;13:59-64

DOI: $10.1159 / 000511203$

Published online: January 28, 2021

(C) 2021 The Author(s)

Published by S. Karger AG, Basel www.karger.com/crn

This article is licensed under the Creative Commons Attribution-NonCommercial 4.0 International License (CC BY-NC) (http://www.karger.com/Services/OpenAccessLicense). Usage and distribution for commercial purposes requires written permission.

\title{
ALS-Like Disorder in Three HIV-Positive Patients: Case Series
}

\author{
Zachary Aaron Satin ${ }^{a} \quad$ Elham Bayat ${ }^{b}$ \\ ${ }^{a}$ George Washington University School of Medicine and Health Sciences, \\ Washington, DC, USA; 'bepartment of Neurology, George Washington University \\ Medical Faculty Associates, Washington, DC, USA
}

\section{Keywords}

Amyotrophic lateral sclerosis · ALS-like syndrome $\cdot$ HIV $\cdot$ Motor neuron disease $\cdot$ HAART

\begin{abstract}
There appears to be a relationship between retroviruses such as HIV and the development of an ALS-like syndrome. Few cases have been reported; however, there exists evidence of a higher frequency of motor neuron disease in HIV-infected patients, as well as potential slowing and reversibility of disease course with combination antiretroviral therapy. We conducted a retrospective chart review of patients presenting to the George Washington University ALS Clinic from September 2006 to June 2018 to identify patients with HIV receiving HAART who were subsequently diagnosed with ALS or an ALS-like disorder. Our goals were to describe our patients' disease course and compare them to general characteristics of ALS. We report three cases of HIV-positive individuals, all male, who were subsequently diagnosed with ALS. Each presented with symptoms of limb onset ALS with involvement of upper and lower motor neurons and whose disease originated at the cervical level. All three had been diagnosed with HIV prior to presentation and were presumably compliant with antiretroviral therapy throughout. Our patients demonstrated effective control of their HIV infection. Each experienced relatively slow progression of motor impairment compared to general ALS characteristics. Our study offers a distinct profile of HIV-positive patients compliant with HAART subsequently diagnosed with an ALS-like disorder. Further study should aim to uncover pathophysiological similarities between motor neuron disease both in the presence and absence of retroviral infection and to develop effective medical therapy for each.




\section{Case Reports in Neurology}

Case Rep Neurol 2021;13:59-64

\begin{tabular}{l|l}
\hline DOI: 10.1159/000511203 & (c 2021 The Author(s). Published by S. Karger AG, Basel
\end{tabular} www.karger.com/crn

Satin and Bayat: ALS-Like Disorder in Three HIV-Positive Patients: Case Series

\section{Introduction}

Amyotrophic lateral sclerosis, or ALS, is a debilitating disorder involving the progressive degeneration of upper and lower motor neurons causing atrophic muscle weakness, eventually precipitating death via respiratory paralysis. ALS is a multisystem disorder that features a combination of oxidative stress, mitochondrial dysfunction, impairment of axonal transport, and other dysfunctional biochemical pathways in its pathogenesis [1, 2]. It has been hypothesized that retroviruses such as HIV-1 may exert selectively toxic effects on motor neurons, thereby generating an ALS-like disorder through proposed mechanisms such as direct neuronal infection, secretion of toxic viral substance, increased immune system reactivity and cytokine secretion, or induction of autoimmune disease [3]. Furthermore, evidence indicates highly active antiretroviral therapy (HAART) may slow and even reverse ALS progression in the HIV-positive population [3-5].

The impact of HIV infection and antiretroviral therapy on the presentation, diagnosis, and progression of ALS is not well defined. Washington, DC has an HIV-positive prevalence of nearly $2 \%$ and the highest annual rate of newly diagnosed HIV infections in the United States [6]. As an academic medical center with an ALS subspecialty clinic in the region, we sought to identify HIV-positive patients who were subsequently diagnosed with ALS or an ALS-like syndrome. In this study, we report three HIV-positive individuals compliant with combination antiretroviral therapy who present with symptoms of spinal onset ALS-like disorder.

\section{Case Report/Case Presentation}

This is a retrospective chart review of patients presenting to the George Washington University ALS Clinic from September 2006 to June 2018. We identified patients with HIV receiving HAART who were subsequently diagnosed with ALS or an ALS-like disorder. Pertinent data collection includes age at symptom onset and ALS diagnosis, time of disease progression, trends in forced vital capacity (FVC), neurological exam scores, and electromyography and nerve conduction study results. We also measured duration of HIV-1 infection, identified trends in CD4 count and HIV viral load, and considered therapeutic approaches implemented. Our objectives were to describe our patients' disease course and compare their symptomology to general characteristics of ALS. During the study period, 322 patients presented to the ALS Clinic; of those, we identified three cases of HIV-positive individuals subsequently diagnosed with ALS. IRB approval for this study was obtained (registration number 180239).

Our patients were males with involvement of both upper and lower motor neurons and whose disease originated at the cervical level. All three were negative for family history of neurodegenerative disorders. Each patient had been diagnosed with HIV prior to ALS diagnosis and was presumably compliant with HAART since their HIV diagnosis. Cases are numbered 1 through 3 based on date of presentation.

Each patient's disease first manifested as distal upper extremity weakness with early atrophy of intrinsic hand muscles. Case 1, a right-handed individual, experienced weakness in the left hand that progressed to involve the entire left upper extremity, followed by contralateral hand weakness 2 years following initial symptom onset. Case 2 , also right-handed, similarly reported distal-to-proximal spread of left upper extremity weakness and muscle atrophy, and after 8 months began to notice motor impairment in the right hand as well. His course is significant for fastest progression to additional extremity involvement of our patients. Case 3 ,

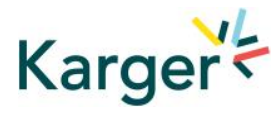




\section{Case Reports in Neurology}

Case Rep Neurol 2021;13:59-64

\begin{tabular}{l|l}
\hline DOI: 10.1159/000511203 & $\odot 2021$ The Author(s). Published by S. Karger AG, Basel
\end{tabular} www.karger.com/crn

Satin and Bayat: ALS-Like Disorder in Three HIV-Positive Patients: Case Series

our only left-handed patient, demonstrated weakness originating in the right intrinsic hand muscles that gradually spread to involve his forearm below the elbow. Yet 14 months after symptom onset, his disease had not extended beyond that point. All three patients denied bulbar symptomology on presentation, though on exam, progressive tongue fasciculation and atrophy were observed in cases 1 and 3. FVC measurements were greater than $80 \%$ predicted at each visit for all three individuals, with the exception of one instance for case 1 at his 12month appointment, during which he was believed to have a mild COPD exacerbation.

Case 1, who had the longest documented disease course, also had the most severe weakness, with $0 / 5$ strength (Medical Research Council Scale) at last visit in left deltoid, biceps, and triceps muscles, and milder weakness in corresponding muscles of the right upper extremity. He initially had diffuse hyperreflexia and positive Babinski, but with time his upper extremity reflexes progressed to $1+$ bilaterally. Case 2 presented exhibiting diffuse hyperreflexia, brisk jaw jerk, positive Hoffmann bilaterally, and positive Babinski on the left. Case 3 had absent Babinski and Hoffman reflexes on presentation, although he demonstrated brisk reflexes in the upper extremities and thus was initially diagnosed with "possible" ALS based on El Escorial criteria. An electromyography for all three cases demonstrated evidence of diffuse acute and chronic denervation and fasciculation in multiple myotomes.

Each patient reported compliance with HAART throughout care at the ALS Clinic. According to trends in CD4 count and viral load, case 1 had the least control over his HIV-infection as evidenced by CD4+ range of 42-150 cells/ $\mathrm{mm}^{3}$ and persistently elevated viral load. He reported limited detail regarding his HIV infection history, unable to recall its duration or his HAART regimen on presentation. Conversely, cases 2 and 3 had 25- and 12-year history of HIV positivity, respectively, and each consistently exhibited CD4 counts within normal limits. Between the two of them, only case 3 had an elevated viral load at any point, measuring 80 copies/mL at an 8-month visit. Cases 2 and 3 supplemented combination antiretrovirals with riluzole. Cases 1 and 2 reported history of cigarette smoking, and case 1 endorsed military service.

\section{Discussion/Conclusion}

Each patient presented with limb as opposed to bulbar onset, and mixed upper and lower motor neuron involvement instead of isolated upper or lower disease, similar to the typical presentation of classical ALS $[2,7,8]$. All three patients presented with nondominant upper extremity weakness. Conversely, Anand et al. [8] report patients with upper extremity onset classic ALS are twice as likely to present with dominant versus nondominant motor impairment. The average age of onset and diagnosis for our cases were 48.3 and 49.7 years, respectively. This is slightly earlier than ALS tends to present in the general population, with symptom onset in the mid-to-late fifties and time from symptom onset to ALS diagnosis approximating 12 months [2].

Our patients experienced relatively slow spread of motor impairment, theoretically the result of ongoing HAART. Case 3 was also a highly athletic individual, likely providing him greater resistance to functional motor impairment. Combination antiretroviral medications may slow advancement and potentially reverse symptomology of an ALS-like syndrome in HIV-infected individuals [4,5]. One study observed motor neuron disease is increasingly less progressive and more responsive to HAART as the time from HIV infection to ALS onset increases [9]. Furthermore, it has been proposed that the HIV virus could mutate to selectively infect motor neurons, offering an explanation for the efficacy of HAART in slowing or

\section{Karger'=}




\section{Case Reports in Neurology}

reversing symptomology, although no direct evidence supports the theory [7]. Klein et al. [10] recently uncovered a shared core protein cluster between ALS and retroviral networks, specifically HIV, Polio, and West Nile, strongly suggesting the likelihood of shared biochemical pathways and offering potential for the retooling of antiviral medications into motor neuron disease therapies. Oxidative stress, mitochondrial dysfunction, impairment of axonal transport, glutamate-driven neurotoxicity, and activation of human endogenous retrovirus $\mathrm{K}$ (HERV-K) represent further examples of dysfunctional mechanisms implicated in the development of ALS, with and without retroviral infection [1,11].

HIV-associated ALS tends to exhibit a male-to-female ratio of 4.8:1, compared to 1.3:1 for ALS and 1:1.5 for HIV [7]. All three of our patients were male; the HIV population in Washington, DC is also majority male, offering an additional explanation for male predilection [6]. There also seems to exist a greater relative frequency of ALS in the HIV-infected population compared to the general population. The annual incidence and prevalence of ALS in the general population are 1-2 and 4-6 per 100,000 people, respectively, with a lifetime risk between $1 / 600$ to $1 / 1,000$ [11]. Comparatively, individuals with HIV have an estimated ALS prevalence of 3.5 per 1,000 HIV-positive individuals and a 27 -fold greater lifetime likelihood of acquiring the disease [7, 12]. Using our three patients weighted against the Washington, DC HIV population in 2018 of 13,003 allows us to estimate an ALS prevalence of 23 per 100,000 for the HIV-positive community [6]. While this estimation is oversimplified, it nonetheless demonstrates the aforementioned increased lifetime risk of developing an ALS-like syndrome if HIV positive.

On its own, ALS is monophasic and irreversible in progression. ALS with concomitant HIV infection, however, demonstrates polyphasic progression and potential reversibility with antiretroviral treatment $[3,4]$. Moreover, some evidence supports specific prognostic factors such as age of onset and diagnosis, site of onset, initial rate of progression, and functional markers such as FVC as having predictive value [13]. FVC is particularly useful as a marker to determine risk of respiratory function. Our cases failed to show significant decrease in FVC throughout their care and therefore exhibited limited respiratory impairment. Still, the phenotypic heterogeneity of ALS makes the establishment of predictive guidelines difficult.

ALS carries an average survival of 3 to 5 years. Factors that may increase likelihood for acquisition include increasing age, endemicity to the Western Pacific, exposure to viral and non-viral toxins such as pesticides, cigarette smoking, military service, and numerous genetic variants $[2,11]$. In the HIV-positive population, HAART remains the most efficacious method of controlling progression. However, there is no definitive cure for ALS. Riluzole decreases glutamate release and therefore reduces neuronal excitotoxicity, providing only a modest lengthening of survival of 3-4 months on average [1]. The more recently FDA-approved edaravone is a neuroprotective drug believed to work as a free radical scavenger and antioxidant, countering oxidative stress-related damage to slow progression of ALS [14]. Other medications merely provide symptomatic relief. Therefore, patients frequently rely on supportive devices including splints, slings, ventilators, cough-assist devices, and electronic scooters.

Although limited in its number of patients, this small case series suggests a distinct profile of HIV-infected patients receiving combination antiretroviral therapy who are subsequently diagnosed with an ALS-like disorder. Due to differences between this patient population and ALS patients without HIV infection, we do not recommend enrolment of these or similar cases in ALS clinical trials, as it may affect the results of the study. We recommend recruitment of a large cohort of this patient population to gain greater understanding of the disorder. Further study should focus on pathophysiological similarities between ALS symptomology with and

\section{Karger'=}




\section{Case Reports in Neurology}

Satin and Bayat: ALS-Like Disorder in Three HIV-Positive Patients: Case Series

without retroviral infection and on development of treatments that slow and potentially reverse progression of the motor neuron disease.

\section{Acknowledgement}

Presented in part at the American Academy of Neurology 71st Annual Meeting, May 4 to May 10, 2019 in Philadelphia, PA. We thank all care team members at the John J. Kelly ALS Clinic at The GW Medical Faculty Associates.

\section{Statement of Ethics}

IRB approval for this study was obtained (registration number 180239). The George Washington University Institutional Review Board approved a Full Waiver of Consent (HRP294) and a HIPAA Full Waiver of Research Subject Authorization (HRP-280) for this study. Patient data is stored in encrypted electronic medical records accessed through the GW Medical Faculty Associates database. At the request of qualified investigators, additional anonymized data not provided in the article will be shared for the purposes of replicating procedures or results.

\section{Conflict of Interest Statement}

The authors have no conflicts of interest to declare.

\section{Funding Sources}

The authors received no funding for the design of this study, or for the collection, analysis, and interpretation of data, or in writing the manuscript.

\section{Author Contributions}

Zachary Satin, BA, is a fourth-year medical student at The George Washington University School of Medicine and Health Sciences. He is responsible for the collection and analysis of data and the generation of the manuscript. Elham Bayat, MD, is an associate professor of neurology and Director of the ALS Clinic at The George Washington University and The GW Medical Faculty Associates. Dr. Bayat is triple board-certified in neurology, electrodiagnostic medicine, and neuromuscular medicine. She is responsible for development of article concept and review of the manuscript.

\section{Karger' ${ }^{\prime \prime}$}




\section{Case Reports in Neurology}

\begin{tabular}{|c|c|}
\hline DOI: 10.1159/000511203 & $\begin{array}{l}\text { (c) } 2021 \text { The Author(s). Published by S. Karger AG, Basel } \\
\text { www.karger.com/crn }\end{array}$ \\
\hline
\end{tabular}

Satin and Bayat: ALS-Like Disorder in Three HIV-Positive Patients: Case Series

\section{References}

1 Mancuso R, Navarro X. Amyotrophic lateral sclerosis: current perspectives from basic research to the clinic. Prog Neurobiol. 2015 Oct;133:1-26.

2 Brown RH, Al-Chalabi A. Amyotrophic Lateral Sclerosis. N Engl J Med. 2017 Jul;377(2):162-72.

3 Moulignier A, Moulonguet A, Pialoux G, Rozenbaum W. Reversible ALS-like disorder in HIV infection. Neurology. 2001 Sep;57(6):995-1001.

4 Verma A, Berger JR. ALS syndrome in patients with HIV-1 infection. J Neurol Sci. 2006 Jan;240(1-2):59-64.

5 Rowland LP. HIV-related neuromuscular diseases: nemaline myopathy, amyotrophic lateral sclerosis and bibrachial amyotrophic diplegia. Acta Myol. 2011 Jun;30(1):29-31.

6 HAHSTA 2018 Annual Report. 2018; Available at: https://dchealth.dc.gov/HAHSTA2018AnnualReport.

7 Alfahad T, Nath A. Retroviruses and amyotrophic lateral sclerosis. Antiviral Res. 2013 Aug;99(2):180-7.

8 Anand KS, Wadhwa A, Garg J, Mahajan RK. Amyotrophic lateral sclerosis-like presentation in a HIV-positive patient [JIAPAC]. J Int Assoc Provid AIDS Care. 2014 Nov-Dec;13(6):515-8.

9 Lorenzoni PJ, Ducci RD, Dalledone GO, Kay CS, de Almeida SM, Werneck LC, et al. Motor neuron disease in patients with HIV infection: report of two cases and brief review of the literature. Clin Neurol Neurosurg. 2018 Aug;171:139-42.

10 Klein JP, Sun Z, Staff NP. Association between ALS and retroviruses: evidence from bioinformatics analysis. BMC Bioinformatics. 2019 Dec;20(S24 Suppl 24):680.

11 Orsini M, Oliveira AB, Nascimento OJ, Reis CH, Leite MA, de Souza JA, et al. Amyotrophic Lateral Sclerosis: New Perpectives and Update. Neurol Int. 2015 Sep;7(2):5885.

12 Bowen LN, Tyagi R, Li W, Alfahad T, Smith B, Wright M, et al. HIV-associated motor neuron disease: HERV-K activation and response to antiretroviral therapy. Neurology. 2016 Oct;87(17):1756-62.

13 Creemers H, Grupstra H, Nollet F, van den Berg LH, Beelen A. Prognostic factors for the course of functional status of patients with ALS: a systematic review. J Neurol. 2015 Jun;262(6):1407-23.

14 Cruz MP. Edaravone (Radicava): A Novel Neuroprotective Agent for the Treatment of Amyotrophic Lateral Sclerosis. P T. 2018 Jan;43(1):25-8. 